Methods We searched the medical literature for studies evaluating performance of dual syphilis/HIV RTs against laboratorybased reference tests for syphilis and HIV, and compared performance across studies. For the syphilis component of the RTs, we compared results using laboratory-based treponemal tests (TPPA or TPHA) as the reference and (when available) $\mathrm{TPPA}+/ \mathrm{RPR}+$ as the reference, considering RPR titers $>1: 4$ to represent active syphilis (vs. previously treated infections).

Results We found 19 studies evaluating dual syphilis/HIV RT performance, of which 7 (37\%) were field evaluations studying at least one of three diagnostics: SD Bioline HIV/Syphilis Duo Test $(\mathrm{n}=4)$; Chembio Dual Path Platform HIV-Syphilis Assay $(\mathrm{n}=2)$; or Medmira Multiplo Rapid TP/HIV Antibody Test $(n=1)$. All used HIV EIA and TPPA or TPHA tests as reference standards; 6 also reported RPR titers. Study populations were pregnant women $(n=3)$, female sex workers $(n=1)$, high-risk men $(n=2)$ and STD clients $(n=1)$, representing a total of 13915 persons (median study size, 415 participants; range 175 - 9983). Across studies, prevalence of HIV ranged from $<1 \%$ to $78 \%$ (median, $25.3 \%$ ), and of T. pallidum (TP) from $<1 \%$ to $40.2 \%$ (median, $8.2 \%$ ). RT sensitivity for HIV against EIA ranged from $93.8 \%$ to $100 \%$ (median, 99.1\%), and specificity from $97 \%$ to $100 \%$ (median, 99.4\%). RT sensitivity for TP against TPPA or TPHA ranged from $52.7 \%$ to $96.5 \%$ (median, 81\%), and specificity from $89 \%$ to $100 \%$ (median, 98.8\%), with better performance in study populations with higher TPPA/RPR + prevalence. Using TPPA+/RPR $+>1: 4$ as the standard, RT sensitivity ranged from $88.5 \%$ to 100\% (median, 94.3\%).

Conclusion In the few published field evaluations of dual syphilis/HIV RTs, performance of the HIV component was high for all tests studied. Sensitivity of the syphilis component against TPPA was poorer, but was more accurate using probable active syphilis infection as the standard.

\section{P1.42 A NEW MAGNETIC PARTICLE-BASED AGGLUTINATION ASSAY FOR ANTI-CARDIOLIPIN ANTIBODY DETECTION IN SYPHILIS}

Mayur Shukla, John W Deutsch, Ellen N Kersh, Yetunde F Fakile. Centres for Disease Control and Prevention, Atlanta, USA

\subsection{6/sextrans-2017-053264.150}

Introduction A magnetic particle based assay was developed for the detection of nontreponemal anti-cardiolipin antibodies in sera of suspected syphilis cases. The presence of this group of antibodies in combination with a reactive treponemal test indicates active syphilis. In this study, we aimed to overcome technical difficulties with attaching cardiolipin to solid support. The newly developed assay potentially offers advantages of better result interpretation, accuracy, and minimum equipment need compared to traditional nontreponemal tests in diagnosing syphilis.

Methods To develop the nontreponemal magnetic agglutination assay (NT-MAA), cardiolipin antigen was modified first through a chemical oxidation process. The oxidised antigen was later covalently linked to magnetic particles. To test the beads, serum samples were mixed with cardiolipin-magnetic particle complex, and incubated in round bottom well microplates. The test was interpreted as reactive when agglutination was observed. Non-reactive sample demonstrated a "button" in the centre of a microwell. The NT-MAA was evaluated using a panel of previously characterised human sera $(n=80)$ and results were compared to rapid plasma reagin (RPR, ASI) and Treponema pallidum particle agglutination tests (TP-PA, Fujirebio). A true positive sample was defined as being reactive for both RPR and TP-PA, while a true negative as both RPR and TP-PA non-reactive.

Results Out of 80 sera tested, 48 were found true positive and 32 true negative with the reference tests. In comparison, the NT-MAA, demonstrated a sensitivity and specificity of $100 \%$ and $96.8 \%$, respectively.

Conclusion Magnetic particle-based assays offer high flexibility because they work with different assay formats. This exploratory study, describes technical advances for development of nontreponemal test (NT-MAA), and also demonstrated an encouraging performance with the studied samples. Additional evaluation with syphilis samples from defined clinical stages of syphilis will help further validate test performance.

\section{P1.43 ABSTRACT WITHDRAWN}

\section{P1.44 MOLECULAR TYPING AND DETECTION OF MACROLIDE RESISTENCE IN TREPONEMA PALLIDUM DNA FROM PATIENTS WITH PRIMARY SYPHILIS IN SÃO PAULO, BRAZIL}

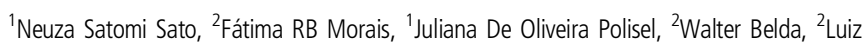
Jorge Fagundes. 'Centro De Imunologia, Instituto Adolfo Lutz, São Paulo - SP, Brazili; ${ }^{2}$ Centro De Saúde Escola Geraldo De Paula Souza, Faculdade De Saúde Pública, USP, São Paulo - SP, Brazil

\subsection{6/sextrans-2017-053264.151}

Introduction Syphilis is a globally occurring sexually transmitted disease caused by Treponema pallidum, a non-cultured in vitro bacterium. Molecular typing of Treponema pallidum strains isolated from patients are useful for investigating the molecular epidemiologic patterns, diversity of strains and antimicrobial resistance patterns. To date, there was no data on the circulating or prevalent subtype in Brazil. In this study we aimed to determine T. pallidum strain diversity and analyse for the mutation associated with macrolide resistance from patients with primary syphilis attended at CSEGPS.

Methods We analysed 24 samples of primary lesion collected from patients attended at CSEGPS between 2013 and 2015. DNA was extracted with DNeasy kit (Qiagen). Standard PCR targeting tpp47 and polA genes was used for screening. Molecular typing was performed by CDC established methods, by determination of the $60 \mathrm{bp}$ repeats within the arp gene, and RFLP analysis of $t p r$ subfamily II genes (E, G and J). Completed by sequence analysis of a variable region of the tp0548 gene. The 23S rDNA mutation was analysed by DNA sequencing of PCR product.

Results: T. pallidum DNA was detected in samples from 15 patients. Among 12 specimes typed, subtype found were $14 \mathrm{~d} / \mathrm{g}$ (6), $14 \mathrm{~d} / \mathrm{d}$ (5) and 12b/d (1). From 10 samples analysed for 23 rDNA mutation, all showed A2058-G, no mutation was detected at A2059. One case presented a different subtype in re-infection. The first was $14 \mathrm{~d} / \mathrm{g}$ and the second was $14 \mathrm{~d} / \mathrm{d}$.

Conclusion: T. pallidum detected in the samples of patients with primary syphilis are of subtypes $14 \mathrm{~d} / \mathrm{g}, 14 \mathrm{~d} / \mathrm{d}$ and $12 \mathrm{~b} / \mathrm{d}$. The macrolide resistance mutation A2058-G was detected in 
all samples analysed. T. pallidum subtyping discriminated reinfection.

\section{P1.45 EVALUATION OF SEKURE RPR REAGENT ON THE SK500 CLINICAL CHEMISTRY SYSTEM}

Kara Osbak, S Abdellati, A Tsoumanis, M Van Esbroeck, T Crucitti, C Kenyon. Institute of Tropical Medicine, Antwerp, Belgium

\subsection{6/sextrans-2017-053264.152}

Introduction An automated and accurate laboratory assay would be of considerable utility to the diagnosis of syphilis and treatment follow-up. We compared the Sekure RPR (Rapid Plasma Reagin) test performed on the SK500 Clinical Chemistry System to RPR card test results.

Methods Serum samples were collected in the context of a 2 year observational cohort study of syphilis infected patients and controls. Syphilis was diagnosed using non-treponemal and treponemal testing. Sera collected at the time of diagnosis (M0) and at 3, 6, 9 and 12 months post-treatment were tested by a Macro-Vue RPR card test (RPR-C) (Becton Dickinson) and a Sekure RPR test (RPR-S) (Sekisui Diagnostics). RPR-S results are expressed in RPR units (R.U.), whereby 1 R.U. equals a 1 -fold change in RPR-C titre. The agreement, linearity and reportable ranges were determined using RPR-C results as the gold standard. Linear regression was used to assess correlations from before and after implementation of an extra dilution step for samples with a strong suspicion of prozone effect.

Results In total, 451 samples from 150 participants were tested, including 120 new syphilis cases and 30 controls. All 30 controls tested negative. Initially there was a weak correlation between RPR-C and RPR-S values $(r=0.15)$. Further analyses identified 72 RPR-S samples with a strong suspicion of prozone effect. We therefore included an extra dilution step (10x) and retested 60/72 samples; values within the expected range were obtained for 58 of them. After implementing the extra dilution step the correlation was moderate $(r=0.61)$, increasing further to $\mathrm{r}=0.91$ for samples with RPR-C titres $\leq 128$. Of the 92 samples that tested RPR-C positive and RPR-S negative, 8 were from M0 (RPR-C: $1-4$ ), which would have led to missed diagnoses.

Conclusion A reasonable correlation was found between the tested methods for mid-range RPR-C results (titre $\leq 128$ ). However, prozone may occur in samples with high antibody concentrations. More investigation is required to elucidate the false negative RPR-S results.

\section{P1.46 COMPARISON OF TWO ENZYME IMMUNOASSAYS FOR THE DETECTION OF IGG AND IGM ANTI-TREPONEMA PALLIDUM ANTIBODIES}

Kara Osbak, S Abdellati, A Tsoumanis, M Van Esbroeck, T Crucitti, C Kenyon. Institute of Tropical Medicine, Antwerp, Belgium

\subsection{6/sextrans-2017-053264.153}

Introduction We aimed to compare two commercial enzyme immunoassays (EIA) for the detection of IgG and IgM antiTreponema pallidum (Tp) antibodies.

Methods Serum samples were collected in the context of a larger study looking at diagnostic biomarkers for syphilis.
Patients with syphilis diagnosed by a treponemal and a nontreponemal assay, were followed for up to two years after treatment. Specimens collected at visit of diagnosis (B), and after three (M3) and six months (M6) of treatment were tested by EIAs detecting anti-Tp IgG and IgM from the manufacturers Euroimmun (EU) and Mikrogen (MI).

Results We tested 338 samples collected from 119 new syphilis cases (23 primary (P), 49 secondary (S), 31 early latent (EL), 16 late latent (LL)) and 30 uninfected controls. A total of 40 participants contributed to 1 sample, 29 to 2 samples and 80 to 3 samples. The controls contributed only to the samples collected at B. Overall 147, 86 and 105 samples were obtained at B, M3 and M6, respectively. The IgM assays were in agreement for $78,1 \%$ of samples; it varied according to the syphilis stage: P: $82,1 \%$; S: $72,5 \%$; EL: $80,8 \%$; LL: $72,1 \%$ and decreased from B to M3: B: 84,4\%; M3: 73,3\%; M6: $73,3 \%$. More samples tested positive with the MI (149) versus the EU (100) $(\mathrm{p}<0.001)$. EU tested all control samples IgM negative, MI reported 1 positive and 1 borderline. The agreement of both $\operatorname{IgG}$ assays was $97,4 \%$; it increased with the stage of infection: P: 91,1\%; S: 97,7\%; EL: 100\%; LL: 100\%, and over time: B: 95,2\%: M3: 98,8\%; M6: 99,0\%. More samples tested positive with the EU (305) versus the MI (300) assay. EU reported all control samples IgG negative, MI detected 1 borderline sample.

Conclusion A good but not perfect agreement was observed for the EIAs detecting IgM. The agreement was highest in primary syphilis and lowest in late latent cases, and decreased over time of treatment. The MI IgM assay reported significantly more positive samples. Overall, we found a good agreement for the EIAs detecting IgG. Albeit that it was somewhat lower for primary syphilis and at baseline.

\section{P1.47 DETECTION OF CERVICAL HUMAN PAPILLOMAVIRUS IN WOMEN ATTENDING FOR CERVICAL CANCER SCRENNING BY VISUAL INSPECTION IN COTE D'IVOIRE}

${ }^{1}$ Ouattara Abdoulaye, ${ }^{2}$ Yeo Alain, ${ }^{2}$ Kouame-Blavo Ehui, ${ }^{3}$ Oura K Pierre, ${ }^{2}$ FayeKette Hortense, ${ }^{2}$ Dosso Mireille. ${ }^{1}$ Felix Houphouet Boigny University, Abidjan - Cote D Ivoire; ${ }^{2}$ Institut Pasteur Cote D'ivoire, Abidjan - Cote D Ivoire; ${ }^{3}$ Hopital General D'abobo Sud Service Gynecoielog, Abidjan - Cote D Ivoire

\subsection{6/sextrans-2017-053264.154}

Introduction Human Papillomaviruses (HPV) are small virus non-enveloped double-stranded circular DNA responsible of genital warts papilloma, precancerous lesions and cancers (cervix, vulva). In Côte d'Ivoire and many lower middle and incomes countries cervical cancer screening program based on visual inspection methods become the gold standard because cytology has shown many limits. This study aims to detect HPV DNA on women attending for cervical cancer screening program based on visual inspection by acid acetic and lugol (IVA/IVL).

Methods From March to December 2015, endocervical secretions from women attending cervical screening by IVA were submitted to HPV determination with PCR. HPV DNA was amplified using PGMY09/11 primers which generated 450 base pairs at the L1 region. The samples harbouring HPV DNA were genotyped using the multiplex PCR with HPV 16, $18,31,33,35,45$ and 51 primers.

Results The medium age of population was 32 years old. On 388 women enrolled in a visual inspection with acetic acid (VIA) program 5.8\% were positif. HPV DNA was obtained in 